\title{
The Anti-Inflammatory Mechanism of Action of a Water Soluble Curcumin Derivative and Mscs in Alzheimer Disease Induced In Rats
}

\author{
MT Abdel Aziz ${ }^{a}$, Ameen Rizk ${ }^{a}$, HH Ahmed $^{a}$, LA Rashed ${ }^{a}$, M Sohier ${ }^{b}$, \\ Ahmed Talaat ${ }^{a}$ and MA ALKaffas ${ }^{a}$ \\ ${ }^{a}$ Department of Medical Biochemistry, Unit of Biochemistry and Molecular \\ Biology, Faculty of Medicine, Cairo University, Cairo, Egypt \\ ${ }^{\mathbf{b}}$ Department of pathology, Faculty of Medicine, Cairo University, Cairo,
} Egypt

\begin{abstract}
Objective: The objective is to evaluate the possible mechanisms of action of a water soluble curcumin derivative and MSCs in Alzheimer disease (AD). Materials and Methods: This work included: Seventy rats which were divided equally into: control group, $A D$ group (induced by lipopolysaccharide), AD group received a novel curcumin derivative (NCD), AD group received pure curcumin, $A D$ group received $M S C$ s then NCD, AD group received MSCs, AD group received MSCs with NCD. Histopathological examination and estimation of serum IL-10 and IL-6 by ELISA, estimation of MDA and GSH in brain tissue by colorimetry and estimation of tissue cholesterol were performed. Results: Histopathological examination of brain tissue from AD animals revealed the presence of plaques. Administration of MSCs or MSCs with NCD into rats after induction of experimental $A D$ improved the histopathological picture with plaque disappearance, while other groups showed decrease of plaque formation. Levels of IL-10 were increased while of IL-6 were decreased in all treated groups. MDA and cholesterol levels were decreased while GSH levels were increased in all treated groups.Conclusion: Administration of BMderived MSCs either alone or with the NCD exert a therapeutic effect on the brain lesion in Alzheimer's disease. This effect may be through the anti-inflammatory and antioxidant action of both MSCs and curcumin. These data suggests that MSCs and curcumin may be a therapeutic target in treatment of $A D$.
\end{abstract}

Key words: Alzheimer disease, Mesenchymal stem cells, Novel curcumin derivative.

\section{INTRODUCTION}

Alzheimer's disease is a progressive, neurodegenerative disease characterized in the brain by abnormal clumps (amyloid plaques) and tangled bundles of fibers (neurofibrillary tangles) composed of misplaced proteins. It manifests by a progressive decline in cognitive abilities such as memory, comprehension and language expression ${ }^{(1)}$.

It was hypothesized that MSCs transplantation may have beneficial effects in $\mathrm{AD}$ patients. Martinet et al. ${ }^{(2)}$ assumed that these effects may be through its anti-inflammatory and antiapoptotic function, via activation of 
microglial cells which eliminate amyloid $\beta$ protein $(\mathrm{A} \beta)$ deposits \& secrete neurotrophic factors, cell proliferation, neuronal differentiation and immunomodulation via suppression of $\mathrm{T}$ cells. Traggiai et al. (3) reported that MSCs inhibit B-cell proliferation and suppress natural killer (NK) cells activation.

Curcumin (1,7bis (4 hydroxy-3 methoxy phenol)-1, 6 heptadiene-3, 5 dione) is a yellow phenolic compound present in turmeric (Curcuma-longa) a widely used spice in Indian cuisine. Curcumin has a number of biological applications along with a significant, anti-inflammatory antioxidant activity both invivo and invitro ${ }^{(4)}$.

Curcumin may affect Alzheimer disease through its anti-inflammatory (reduce inflammatory cytokines) and anti-oxidant function (reduce lipid peroxidation $)^{(5)}$. Hong et al. ${ }^{(6)}$ reported that curcumin lowered plasma and tissue cholesterol and reduced $\mathrm{A} \beta$ levels and plaque burden. Others proved that curcumin markedly inhibited Tau phosphorylation (7), increased level of neurotrophic factor and increased transcription of Toll Receptors (TLRs) genes. Fiala et al. ${ }^{(8)}$ have reported that downregulation of genes such as toll like receptors in $\mathrm{AD}$ patients is related to insufficient clearance of $A \beta$ from the brain).

Studies over the past three decades related to absorption, distribution, metabolism and excretion of curcumin have revealed poor absorption and rapid metabolism of curcumin that severely curtails its bioavailability ${ }^{(9)}$. Because of the poor bioavailability of pure curcumin, a new water soluble curcumin derivative (Patent pending
PCT/EG2010/000008) was used in this study.

This work aims to study the possible mechanisms of action of a water soluble curcumin derivative and MSCs in Alzheimer disease.

\section{MATERIALS \& METHODS}

This work was performed at the Unit of Biochemistry and Molecular Biology at The Medical Biochemistry Department, Faculty of Medicine, Cairo University, Cairo, Egypt.

A novel water soluble curcumin derivative (NCD) was developed through covalent modification of the curcumin molecule on sites remote from its natural functional groups. The NCD was presented free of charge to the participating researchers as a personal non-profit scientific participation in the present study. The novel derivative with $53.2 \%$ curcumin content is registered as international patent protected by the rights of "The Patent Cooperation Treaty" and is the personal property of its inventors (PCT/EG2008/000044, WO 2010/057503, Regional phase European Patent Application No. 08878223) ${ }^{(\mathbf{1 0})}$.

\section{Preparation of the animal model Experimental animals}

This study included seventy female rats obtained from an inbred strain of matched age and weight (6 months-1 year \& 120-150gm). Animals were inbred in the Experimental Animal Unit, Faculty of Medicine, Cairo University. Rats were bred and maintained in an air conditioned animal house with specific pathogen free conditions, and were subjected to a 12:12-h daylight/darkness. Animals 
were fed a semi-purified diet that contained (gm/kg): 200 casein, 555 sucrose, 100 cellulose, 100 fat blends, 35 vitamin mix, and 35 mineral mix. All animal experiments received approval from the Institutional Animal Care Committee. They were divided into 7 groups as follow:

Group 1 (AD): Ten rats as a positive control group (induced by Lipopolysaccharide (LPS) that was given as a single injection at a dose of $0.8 \mathrm{mg} / \mathrm{kg}$, intraperitoneally every week for 3 weeks) ${ }^{(11)}$.

Group 2 (control): Ten rats as a negative control group (normal healthy rats).

Group 3 (NCD): Ten induced Alzheimer rats that received curcumin derivative only $(150 \mathrm{mg} / \mathrm{kg}$, body weight, orally daily for 3 weeks).

Group 4 (Pure): Ten induced Alzheimer rats that received pure curcumin $(80 \mathrm{mg} / \mathrm{kg}$, body weight, orally daily for 3 weeks) ${ }^{(\mathbf{1 2})}$.

Group 5 (MSCs then NCD): Ten induced Alzheimer rats that received MSCs (which were processed and cultured for 14 days, in a single dose of $10^{6}$ cells per rat ${ }^{(13)}$, given by Intravenous infusion at the rat tail vein for one Month), then $\mathrm{NCD}(150 \mathrm{mg} / \mathrm{kg}$, body weight, orally daily for 3 weeks) two weeks later.

Group 6 (MSCs): Ten induced Alzheimer rats that received intraperitoneal MSCs only.

Group 7 (MSCs+NCD): Ten induced Alzheimer rats that received MSCs ( which were processed and cultured for 14 days, in a single dose of $10^{6}$ cells per rat (13), given by Intravenous infusion at the rat tail vein for one Month)and NCD (150 mg/kg, body weight, orally daily for 3 weeks).
At the planned time (30 days), venous blood was collected from the retro-orbital vein from rats of all groups, left to clot for $30 \mathrm{~min}$., centrifuged at $10,000 \mathrm{rpm}$ for $20 \mathrm{~min}$. then separated, serum was kept frozen till analysis of IL-10, IL-6 by ELISA. Animals were sacrificed by anesthesia, and brain tissue was harvested for assessment of the following:

1. Estimation of serum levels IL-10, IL-6 by ELISA.

2. Estimation of Malondialdehyde (MDA) and Reduced glutathione (GSH) in rat brain by colorimetry.

3. Estimation of brain tissue cholesterol.

4. Histopathological examination of brain tissues.

I. Isolation, propagation, identification and labeling of $\mathrm{BM}$ derived MSCs from rat

\section{A) Isolation and propagation of BM- derived MSCs from rats}

Bone marrow was harvested by flushing the tibiae and femurs of 6week-old male white albino rats with Dulbecco's modified Eagle's medium (DMEM, GIBCO/BRL) supplemented with $10 \%$ fetal bovine serum (GIBCO/BRL). Nucleated cells were isolated with a density gradient [Ficoll/Paque (Pharmacia)] and resuspended in complete culture medium supplemented with $1 \%$ penicillin-streptomycin

(GIBCO/BRL). Cells were incubated at $37^{\circ} \mathrm{C}$ in $5 \%$ humidified $\mathrm{CO}_{2}$ for 12 14 days as primary culture or upon formation of large colonies. When large colonies developed (80-90\% confluence), cultures were washed twice with phosphate buffer saline (PBS) and the cells were trypsinized with $0.25 \%$ trypsin in $1 \mathrm{mM}$ EDTA 
(GIBCO/BRL) for $5 \mathrm{~min}$ at $37^{\circ} \mathrm{C}$. After centrifugation, cells were resuspended in serum-supplemented medium and incubated in $50 \mathrm{~cm}^{2}$ culture flask (Falcon). The resulting cultures were referred to as firstpassage cultures ${ }^{(\mathbf{1 4})}$. MSCs in culture were characterized by their adhesiveness and fusiform shape.

B) Identification of BM- derived MSCs from rat

Cells were identified as being MSCs by their morphology, adherence, and their typical fusiform appearance (15) .CD29 gene expression was detected by RT-PCR as a marker of MSCs ${ }^{(16)}$.

\section{C) Labeling of MSCs with PKH26}

MSCs were harvested during the $4^{\text {th }}$ passage and were labeled with PKH26, which is a red fluorochrome. The fluorochrome has excitation $(551 \mathrm{~nm})$ and emission $(567 \mathrm{~nm})$ characteristics compatible with rhodamine or phycoerythrin detection systems. The linkers are physiologically stable and show little to no toxic side-effects on cell systems. Labeled cells retain both biological and proliferating activity, and are ideal for in vitro cell labeling, in vitro proliferation studies and long, in vivo cell tracking. In the current work, MSCs were labeled with PKH26 from Sigma Company (St. Louis, MO/USA). Cells were centrifuged and washed twice in serum free medium. Cells were pelleted and suspended in dye solution. Cells were injected intravenously into rat tail vein. After one month, brain tissue was examined with a fluorescence microscope to detect and trace the cells.

\section{I- Estimation of the serum levels of} IL-10 and IL-6 by ELISA

Serum IL-10 and IL-6 were assayed by commercially available Enzyme-linked immunosorbent assays (ELISA) kits supplied by Q \&D system Quatin USA.

II-Estimation of the levels of MDA and GSH in brain tissue by colorimetry:

Brain tissue MDA was assayed by a commercial kit supplied by Biodiagnostic, Egypt ${ }^{(17)}$. Brain tissue GSH was assayed by a commercial kit supplied by Biodiagnostic, Egypt ${ }^{(18)}$.

\section{III-Estimation of Tissue cholesterol}

Brain tissue was homogenized and centrifugated at $3000^{\circ} \mathrm{C}$ for 10mins.Brain cholesterol was measured by using a commercially available kit supplied by Diamond Diagnostics, Egypt ${ }^{(19)}$.

IV-Histopathological examination of brain tissues

Autopsy samples were taken from the brain of rats in different groups and fixed in $10 \%$ formol saline for twenty four hours. Washing was done in tap water then serial dilutions of alcohol (methyl, ethyl and absolute ethyl) were used for dehydration. Specimens were cleared in xylene and embedded in paraffin at 56 degree in hot air oven for twenty four hours. Paraffin bees wax tissue blocks were prepared for sectioning at 4 microns thickness by slidge microtome. The obtained tissue sections were collected on glass slides, deparaffinized, stained by hematoxylin \&eosin stain for routine examination then examination was done through the light electric microscope ${ }^{(20)}$. 


\section{Statistical analysis}

Data were coded and entered using the statistical package SPSS version 16. Data was presented as mean \pm standard deviation. Comparisons between groups were done using analysis of variance (ANOVA) with multiple comparisons post hoc test in normally distributed quantitative variables while non para metrical kruscal-wallis test and mann-whitney test were used for non-normally distributed quantitative variables. Pvalues less than 0.05 were considered statistically significant.

\section{RESULTS}

\section{I-MSCs culture, identification \& homing}

Isolated and cultured undifferentiated MSCs reached 70-80 \% confluence in 14 days. Typical fusiform appearance in culture (Figure 1). MSCs labeled with PKH26 fluorescent dye were detected in the brain tissues confirming that these cells homed into the brain tissues (Figure 3). In addition MSCs were identified by surface marker CD29 (+) by RT-PCR as a marker of MSCs (Figure 2).

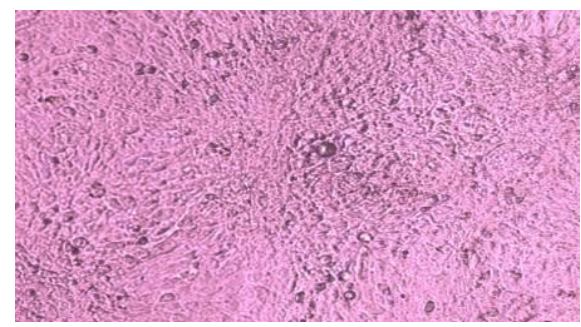

Figure (1): A: MSCs in culture.

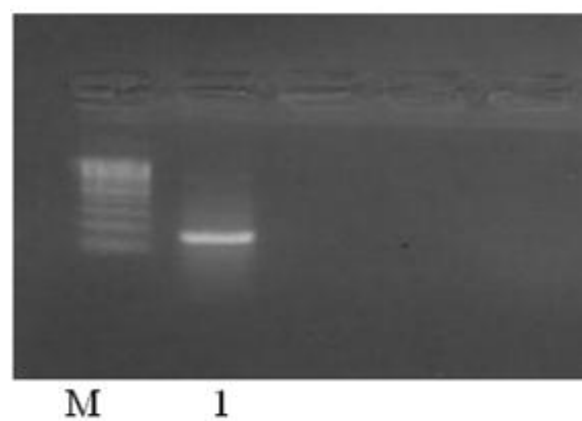

Figure (2): An agarose gel electrophoresis shows PCR products of CD29 gene expression in MSC culture (261 bp) (as a molecular marker for rat MSCs)

Lane M : DNA marker with $100 \mathrm{bp}$.

Lane 1 : MSCs culture.

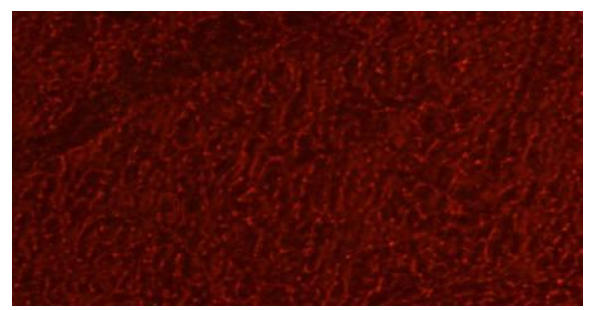

Figure (3): Labeling of MSCs with PKH26 fluorescent dye detected in the brain tissue, confirming that these cells homed into the brain tissue.

\section{II-Estimation of Serum levels of IL- 10 and IL-6 by ELISA (Figure 4)}

- There was a significant decrease $(\mathrm{p}<0.001)$ in the mean IL-10 level $(199.74 \pm 14.11)$ and a significant increase $(\mathrm{p}<0.001)$ in the mean IL6 level $(128.51 \pm 9.08)$ in the AD group compared to control groups $(415.99 \pm 6.12$ and $38.95 \pm 5.3)$, respectively. 
- There was a significant increase $(p<0.001)$ in the mean IL-10 level in the $\mathrm{AD}$ group receiving the NCD (311.07 \pm 10.68$)$, AD group receiving pure curcumin (263.51 13.15$)$, the AD group receiving MSCs then NCD (365.38 \pm 15.81$)$, the $\mathrm{AD}$ group receiving MSCs $(364.33 \pm 17)$, and the AD group receiving MSCs+NCD (397.71 14.23$)$ when compared to AD group (199.74 \pm 14.11$)$.

- On the other hand there was a significant decrease $(\mathrm{p}<0.001)$ in the mean IL-6 level in the AD group receiving the NCD (74.48 \pm 8.72$)$, AD group receiving pure curcumin $(95.84 \pm 4.06)$, the $\mathrm{AD}$ group receiving MSCs then NCD (62.56 \pm 11.78$)$, the AD group receiving MSCs (75.64 \pm 5.35$)$, and the $\mathrm{AD}$ group receiving

$\mathrm{MSCs}+\mathrm{NCD}$ (56.32 \pm 8.24$)$ when compared to AD group (128.51 \pm 9.08$)$.

- On comparing the effect of oral administration of NCD and pure curcumin on IL-10 and IL-6 levels in the AD, NCD significantly decreased IL-10 and significantly increased IL-6 levels more than pure curcumin thus providing more effective results than pure curcumin.

- Our results reported that when AD rats received MSCs, there was a significant increase in the IL-10 level compared to the $\mathrm{AD}$ rats who received the oral NCD. However, no significant difference was detected in IL-6 levels in both groups.

- The mean IL-10 level in the AD group receiving concomitant MSCs +NCD (397.71 \pm 14.23$)$ showed a significant increase $(p<0.001)$ when compared to the $\mathrm{AD}$ group receiving $\mathrm{MSCs}$ followed by NCD $(365.38 \pm 15.81)$ and the $\mathrm{AD}$ group receiving MSCs only $(364.33 \pm 17)$. On the other hand, the mean IL-6 level in the $\mathrm{AD}$ group receiving concomitant MSCs +NCD (56.32 \pm 8.24$)$ showed a significant decrease $(p<0.001)$ when compared to the AD group receiving MSCs (75.64 \pm 5.35$)$, while no significant difference $(p=.578)$ was detected when compared to the $\mathrm{AD}$ group receiving MSCs followed by NCD (62.56 \pm 11.78$)$. 


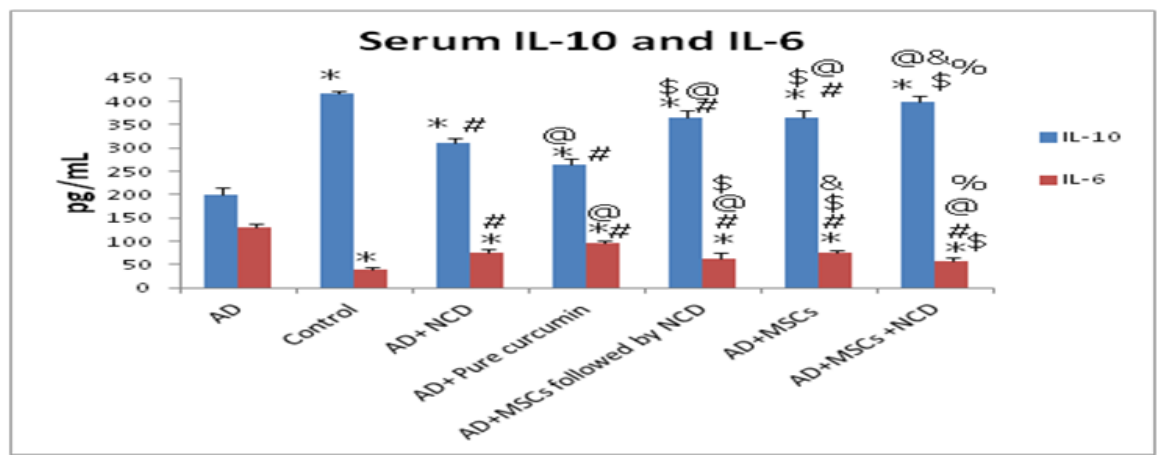

* statistically significant compared to corresponding value in $A D$ group $(P<0.05)$

\# statistically significant compared to corresponding value in control group $(\mathrm{P}<0.05)$

@statistically significant compared to corresponding value in NCD group $(\mathbf{P}<0.05)$

$\$$ statistically significant compared to corresponding value in pure curcumin group $(\mathbf{P}<0.05)$

\& statistically significant compared to corresponding value in MSCs then NCD group $(\mathrm{P}<0.05)$

$\%$ statistically significant compared to corresponding value in MSCs group $(\mathbf{P}<0.05)$

Figure (4): Comparison between the serum IL-10 and IL-6 levels in studied groups.

\section{III-Estimation of MDA and GSH by colorimetry (Figure 5)}

- Our results showed a significant $(p<0.001)$ increase in the mean MDA level $(27.08 \pm 3.27)$ and a significant $(\mathrm{p}<0.001)$ decrease in the mean GSH level in the AD group (15.38 \pm 2.05$)$ compared to the control groups $(9.36 \pm 0.92$ and $49.32 \pm 6.63)$, respectively.

- On comparing the effect of oral administration of NCD and pure curcumin on MDA and GSH levels in the AD, NCD significantly increased $(\mathrm{p}<0.001)$ the GSH level compared to the pure curcumin while no significant difference $(p=0.960)$ was detected in MDA levels.

- The administration of MSCs to the $\mathrm{AD}$ group resulted in a nonsignificant difference $(p=0.603)$ in the levels of MDA and GSH $(\mathrm{p}=.879)$ compared to the $\mathrm{AD}$ group receiving the NCD.

- Our results showed that the concomitant injection of MSCs and oral administration of NCD or the injection of MSCs followed 2 weeks later by NCD to AD rats showed a non significant difference $\quad(p=.999 \quad$ and $\mathrm{p}=0.558$ ), respectively in MDA levels when compared to the $\mathrm{AD}$ rats injected with MSCs. On the other hand, the mean GSH level in the $\mathrm{AD}$ group receiving MSCs $+\mathrm{NCD}(41.51 \pm 2.26)$ showed a significant increase $(\mathrm{p}<0.05)$ when compared to the $\mathrm{AD}$ group receiving MSCs (34.37 \pm 3.49$)$ and to the AD group receiving MSCs then NCD (33.42 \pm 3.25$)$. 


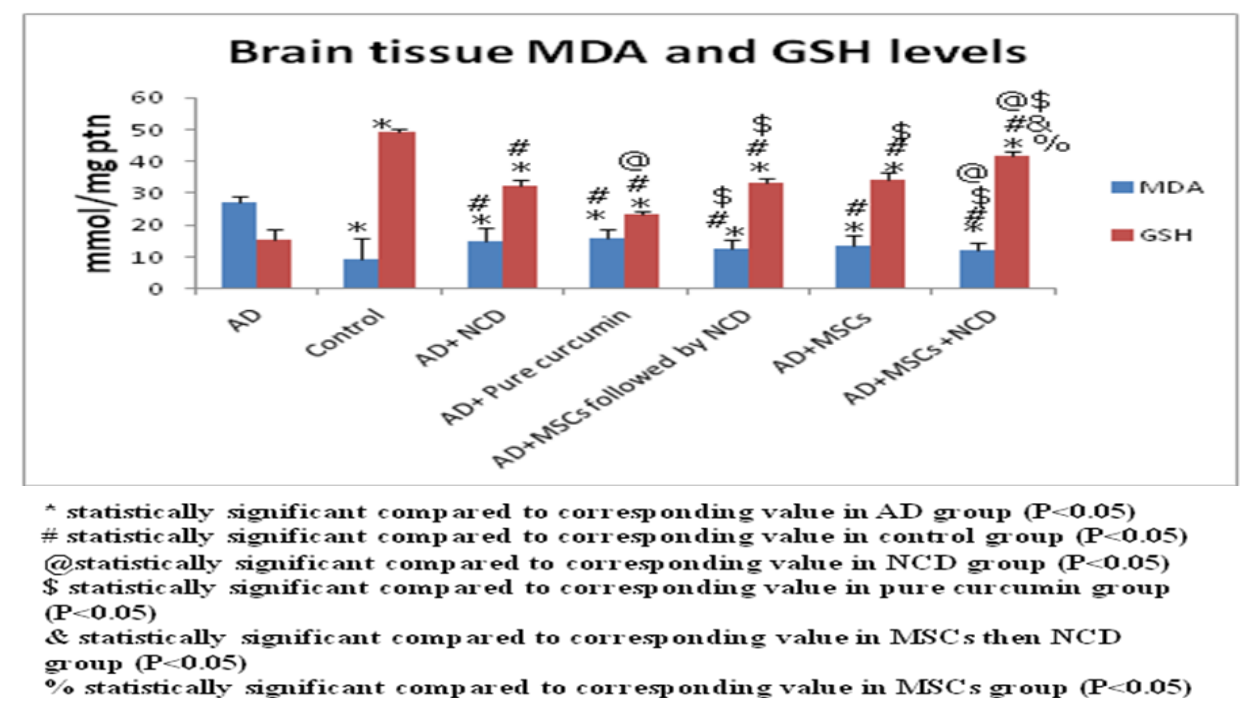

Figure (5): Comparison between the MDA and GSH levels in rat brain of the studied groups.

\section{IV- Estimation of Tissue cholesterol (Figure 6)}

- There was a significant increase $(p<0.001)$ in the mean cholesterol level in the $\mathrm{AD}$ group $(51.84 \pm 5.14)$ compared to control group (20.05 \pm 2.87$)$.

- There was a significant decrease $(p<0.05)$ in the mean cholesterol level in the $\mathrm{AD}$ group receiving NCD $(30.93 \pm 1.81)$, AD group receiving pure curcumin (42.56 \pm 2.37$)$, the $\mathrm{AD}$ group receiving MSCs then NCD (24.59 \pm 9.13$)$, the AD group receiving MSCs (31.6 \pm 8.25$)$, and the $\mathrm{AD}$ group receiving $\mathrm{MSCs}$ $+\mathrm{NCD}(28.38 \pm 8.14)$ as compared to $\mathrm{AD}$ group (51.84 \pm 5.14$)$.

- On comparing the effect of oral administration of NCD and pure curcumin on cholesterol levels in the AD group, NCD significantly decreased $\quad(\mathrm{p}<0.001) \quad$ the cholesterol level $(30.93 \pm 1.81)$ compared to the pure curcumin (42.56 \pm 2.37$)$.

- The mean cholesterol level in the AD group receiving MSCs $(31.6 \pm 8.25)$ showed no significant difference $(\mathrm{p}=1.000)$ when compared to the $\mathrm{AD}$ group receiving the NCD $(30.93 \pm 1.81)$

- The mean cholesterol levels in the $\mathrm{AD}$ group receiving $\mathrm{MSCs}+\mathrm{NCD}$ $(28.38 \pm 8.14)$ showed an insignificant difference when compared to the $\mathrm{AD}$ group receiving MSCs $(\mathrm{p}=0.899)$ (31.6 \pm 8.25$)$ and to the $\mathrm{AD}$ group receiving MSCs then NCD $(\mathrm{p}=0.806)(24.59 \pm 9.13)$. 


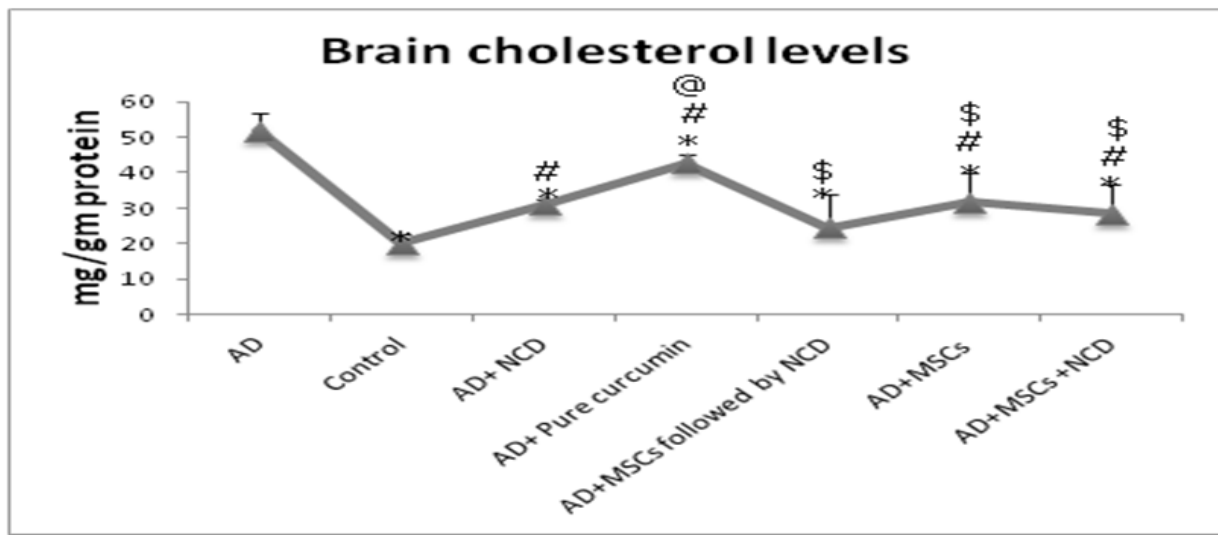

* statistically significant compared to corresponding value in AD group (P<0.05)

\# statistically significant compared to corresponding value in control group (P<0.05)

@statistically significant compared to corresponding value in NCD group (P< $<05)$

$\$$ statistically significant compared to corresponding value in pure curcumin group $(\mathbf{P}<\mathbf{0 . 0 5})$

\& statistically significant compared to corresponding value in MSCs then NCD group $(\mathbf{P}<\mathbf{0 . 0 5})$

$\%$ statistically significant compared to corresponding value in MSCs group (P<0.05)

Figure (6): Comparison between the cholesterol levels in rat brain of the studied groups.

\section{VI-Histopathological examination of brain tissues in different groups}

- Histopathological results of rat brain control group showed intact histological structure of the hippocampus (Figure 7).

- Histopathological examination of the brain tissue of the AD group showed focal areas of plaque formation and gliosis in the striatum of the cerebrum (Figure 8). AD received NCD group: Focal areas of plaque formation with diffuse gliosis in between (Figure 9), AD received pure curcumin group: Focal areas of plaque formation in the striatum of the cerebrum (Figure 10).AD received MSCs then NCD group: The striatum of the cerebrum showed focal plaques formation (Figure 11), AD received MSCs group: Focal hemorrhage was detected in the hippocampus (Figure 12). AD received MSCs and NCD group: Mild diffuse gliosis was detected in the hippocampus (Figure 13).

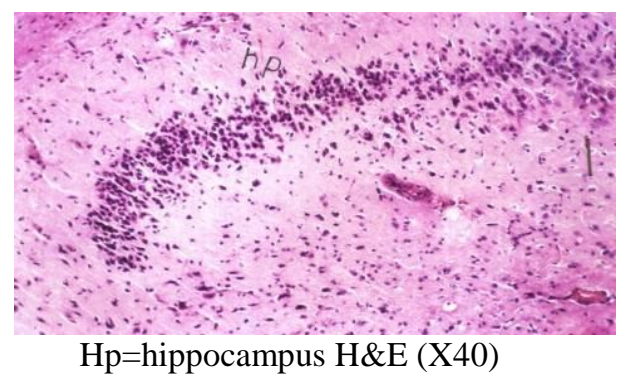

Figure (7): Histopathological examination of control group 


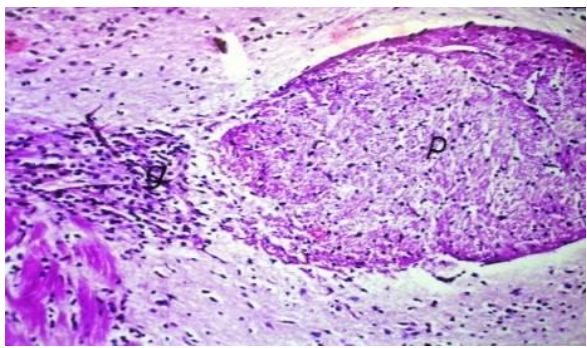

H\&E (X40)

Figure (8): Histopathological examination of rat brain of induced Alzheimer model

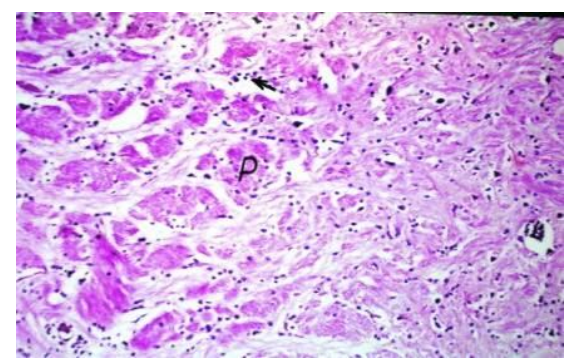

H\&E (X40)

Figure (9): Histopathological examination of rat brain of $\mathrm{AD}$ that received NCD.

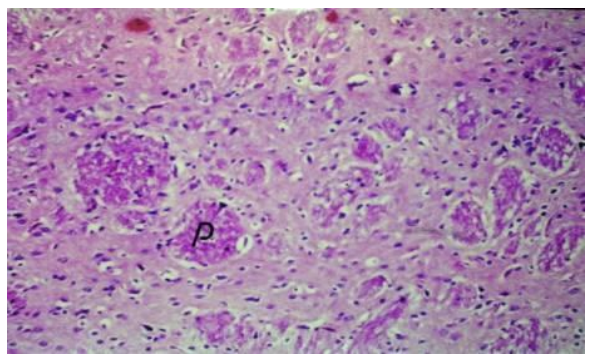

H\&E (X40)

Figure (10): Histopathological examination of rat brain of $\mathrm{AD}$ that received pure curcumin .

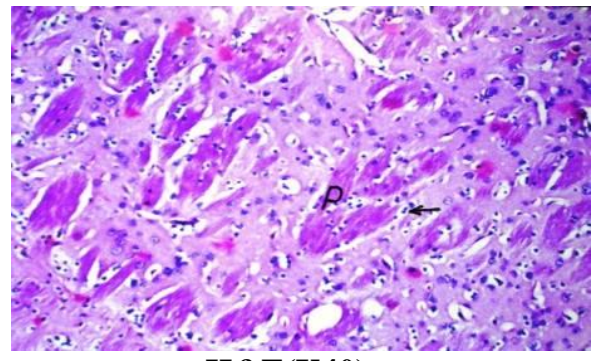

H\&E(X40)

Figure (11): Histopathological examination of rat brain of AD that received MSCs then NCD.

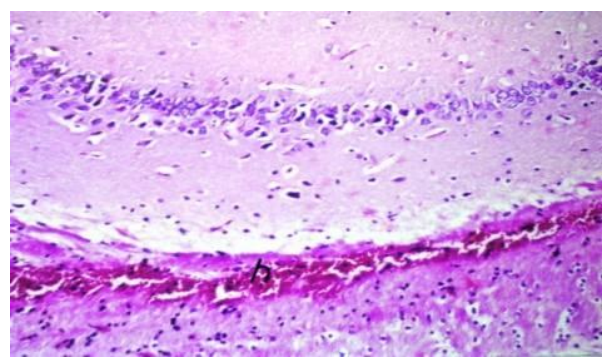

H\&E (X40)

Figure(12):Histopathological examination of rat brain of $\mathrm{AD}$ that received MSCs.

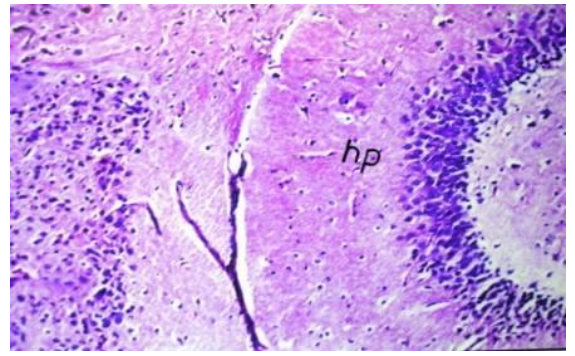

H\&E (X40)

Figure (13): Histopathological examination of rat brain of $\mathrm{AD}$ that received MSCs and NCD. 


\section{DISCUSSION}

Alzheimer's disease (AD) is a neurodegenerative disease that leads to atrophy throughout the brain including the basal forebrain cholinergic system, amygdala, hippocampus and several other cortical areas. $\beta$-amyloid plaques and tau neurofibrillary tangles are hallmark features of $\mathrm{AD}{ }^{(21)}$. Current therapies are limited to provide only partial and temporary improvement of AD symptoms ${ }^{(22)}$.

Stem cell therapy holds a great promise for the repair of injured tissues and organs, including the brain due to the unique ability to differentiate and self renewal ${ }^{(23)}$. Curcumin may be a promising therapy for $\mathrm{AD}$ because it has different neuroprotective activities, including antioxidant, antiinflammatory and antiamyloidogenic properties (24). One of the reasons suggested for the lack of beneficial results of curcumin in $\mathrm{AD}$ clinical trials has been the inability to produce sufficient brain levels following oral absorption ${ }^{(25)}$. It has been repeatedly shown that curcumin has poor water solubility and poor oral bioavailability, and that much of an administered dose is excreted in the feces ${ }^{(\mathbf{9})}$. For this reason, we used in our study a novel curcumin derivative (NCD) to improve the bioavailability and delivery of curcumin.

In the present study, we aimed to evaluate the possible mechanisms of action of a novel curcumin derivative and MSCs in Alzheimer disease.

Brain inflammation is a pathological hallmark of AD. Inflammation clearly occurs in pathologically vulnerable regions of the $\mathrm{AD}$ brain, with increased expression of acute phase proteins and proinflammatory cytokines which are hardly evident in the normal brain ${ }^{(26)}$.

In the present work, administration of stem cells alone or with NCD or pure curcumin significantly increased mean IL-10 and decreased mean IL-6 levels in $\mathrm{AD}$ rats and NCD gave significantly better results compared to pure curcumin.

These results agree with Friedman (27) who reported that curcumin in low doses enhanced stem cell proliferation in vitro and proved that curcumin acts synergistically with stem cells in a mouse model of spinal cord injury .They have documented antioxidant and anti-inflammatory effects of micromolar concentrations of curcumin in cultured tumor cell lines as well as normal non-neuronal cells.

Mesenchymal stem cells (MSCs) have the inherent ability to migrate to multiple organs and to exert immunosuppressive activity. Inflammatory arthritis model was injected weekly three times with IL-10MSCs 21 days after primary immunization. Cytokine assay was done by ELISA.The control mice received vehicle or MSCs alone and the results showed that cultured IL-10MSCs were able to secrete high amounts of IL-10 in vitro. Serum IL-6 level was also decreased by the administration of IL-10-MSCs. In contrast, spleen cells of IL-10-MSCtreated mice produced higher amounts of IL-4 than those of control mice. Interestingly, although not as potent as IL-10-MSCs, injection of naive MSCs alone decreased serum levels of IL-6 (28).

MSCs caused mature dendritic cells (DCs) type 1 (DC1) to decrease 
tumor necrosis factor $\alpha$ (TNF- $\alpha)$ secretion and mature DC2 to increase interleukin-10 (IL-10) secretion; MSCs caused Th1 cells to decrease interferon $\gamma(\mathrm{IFN}-\gamma)$ and caused the Th2 cells to increase secretion of IL-4; MSCs caused an increase in the proportion of regulatory $\mathrm{T}$ cells (Treg) to more tolerant phenotype and decrease secretion of IFN- $\gamma$ from the natural killer (NK) cells ${ }^{(29)}$.

Dysregulation of cholesterol homeostasis in the brain has been linked to chronic neurodegenerative disorders, including Alzheimer's disease (AD), Huntington's disease (HD), Parkinson's disease (PD), Niemann-Pick type C (NPC) disease and Smith-Lemli Opitz syndrome (SLOS), as well as to acute neuronal injuries such as stroke and brain trauma ${ }^{(30)}$.

In our work there was a significant increase in the mean brain tissue cholesterol in the AD group compared to control group.This agrees with Searcy (31) who observed that individuals with AD pathology have higher serum levels of total cholesterol as well as LDL. Hypercholesterolemia may actually lead to cholesterol enrichment of microdomains within the cell membrane, favoring APP cleavage, and subsequent increased production via $\gamma$-secretases located within these domains. It is thought that high levels of cholesterol would favor the cleavage of the APP by the beta and gamma secretases, which would produce the $A \beta 40$ or $A \beta 42$ peptides, which are thought to be responsible for AD pathology.

In our study, when the NCD or pure curcumin was orally administered to $\mathrm{AD}$ rats, there was a significant decrease in brain cholesterol levels compared to the AD group.This decrease was more significant in rats receiving NCD.

Tian et al. ${ }^{(32)}$ found that chronic cerebral hypoperfusion has been well depicted as a common pathological status contributing to neurodegenerative disease such as Alzheimer's dementia. Cholesterol is critical to brain growth, but high levels of cholesterol have been associated with neurogenerative disease.

Dysregulation of cholesterol homeostasis in $\mathrm{AD}$ might be due to the increased expression of Caveolin1 (Cav1) gene leading to alterations of cholesterol distribution in the $\mathrm{AD}$ brain. Dong et al. ${ }^{(33)}$ also showed that in aged rats after administration of 6and 12-week curcumin-fortified diets, that Cav1 is involved in spatial memory formation and synaptic plasticity.

Antioxidant enzymes are important in preventing an excessive accumulation of ROS. Membrane lipids present in subcellular organelles are highly susceptible to free radical damage. Malondialdehyde (MDA) is a by-product of lipid peroxidation induced by free radicals and is also widely used as a biomarker of oxidative stress ${ }^{(34)}$.

In the present study, there was a significant decrease in the mean GSH and a significant increase the MDA levels in the $\mathrm{AD}$ group compared to control group. Gustaw-Rothenberg et al. (35) and Pan et al. (34) showed similar results.

Our results agree with Eldenshary et al. ${ }^{(36)}$ who reported that administration of MSCs significantly decreased serum LDH, MDA and 
MPO enzymes and significantly increased GSH in the MSCs group in an experimental model of arthtritis compared to the control group.Also, when curcumin $(5,15,45$ $\mathrm{mg} / \mathrm{kg}$ ) was injected intraperitonealy (i.p.) once daily for a period of 10 days, it was able to decrease MDA and SOA levels significantly and improve learning and memory in rats of ${ }^{(37)}$.

Curcumin has been demonstrated to have a strong antioxidant neuroprotective effects, scavenging ROS and neutralizing nitric-oxide(NO-) based free radicals. The antioxidant activity of curcumin may be mediated through antioxidant enzymes such as superoxide dismutase (SOD), catalase (CAT), and glutathione peroxidase (GSH-Px). Curcumin has been shown to serve as an acceptor, reacting with glutathione (GSH) and thioredoxin. Depletion in cellular GSH levels is an important measure of oxidative stress, which is implicated in the pathogenesis of AD. A study on postmortem brain of $\mathrm{AD}$ patients has revealed decreased levels of GSH in some area of the brain. It was demonstrated that curcumin is able to replenish the intracellular GSH pool by changing the nuclear content and/or activation of specific transcription factors. It was reported that curcumin mediates the direct detoxification of reactive nitrogen species such as peroxynitrite, thus exerting an antioxidant activity ${ }^{(24)}$.

Our histopathological results agree with the work of Ebrahimi et al. ${ }^{(38)}$ who reported that upon transplantation into the brain, MSCs promote endogenous neuronal growth, decrease apoptosis, reduce levels of free radicals, encourage synaptic connection from damaged neurons and regulate inflammation. MSCs may encourage repair and new growth of neurons through providing neurotrophic factors (BDNF), which seems to be a significant aspect among the mechanisms of MSCs for the treatment of brain diseases.

Our results also agree with Lee et al. ${ }^{(13)}$ who strongly suggested that intracerebral BM-MSC transplantation not only reduces amyloid load and tau phosphorylation in the brain, but also prevents cognitive decline and memory impairment, this occurs by activation of an endogenous microglial population with an alternative phenotype that has neuroprotective effects.

In addition, BM-MSC transplantation can reduce tau hyperphosphorylation in a pattern similar to aggregated A $\beta$. BM-MSC treatment ameliorates spatial learning and memory impairments associated with the accumulation of $\mathrm{A} \beta$ peptide as with BM-MSCs performed significantly better on the water maze test than PBS-treated counterparts ${ }^{(39)}$.

Our work is in accordance with recent advances in a study of $\mathrm{AD}$ performed by Wang et al. ${ }^{(5)}$ who revealed that astrocytes (AS) are key factors in the early pathophysiological changes in AD. Glial fibrillary acidic protein (GFAP), a marker specific to $\mathrm{AS}$, is markedly more manifest during morphological modifications and neural degeneration signature during the onset of AD. Several studies investigating the functionality of curcumin have shown that it not only inhibits amyloid sedimentation but also accelerates the disaggregation of amyloid plaque. 
Conclusion: Administration of BM-derived MSCs either alone or with the NCD exerts a therapeutic effect on the brain lesion in Alzheimer's disease. This effect may be through the antiinflammatory and antioxidant action of both MSCs and curcumin.

\section{REFERENCES}

1. Brookmeyer R, Johnson E, Ziegler - Graham K ,Arrighi HM.(2007): Forecasting the global burden of Alzheimer's disease. Alzheimers Dementia.; 3:186-191.

2. Martinet L, Fleury-Cappellesso S, Gadelorge $M$, Dietrich G, Bourin P, Fournie JJ, Poupot R (2009):A regulatory cross-talk between Vgamma9Vdelta2 T lymphocytes and mesenchymal stem cells. Eur. J. Immunol., 39(3): 752-62.

3. Traggiai E, Volpi S, Schena F, Gattorno M, Ferlito F, Moretta L, Martini A (2008): Bone marrow-derived mesenchymal stem cells induce both polyclonal expansion and differentiation of $\mathrm{B}$ cells isolated from healthy donors and systemic lupus erythematosus patients. Stem Cells 26(2): 562569.

4. Deng G, Yu JH, Ye ZQ, Hu ZQ (2008): Curcumin inhibits the expression of vascular endothelial growth factor and androgenindependent prostate cancer cell line PC-3 in vitro. Zhonghua Nan Ke Xue.; 14:116-121.

5. Wang SL, Li Y, Wen Y, Chen YF, Na LX, Li ST, Sun CH.(2009): Curcumin, a potential inhibitor of up-regulation of TNFalpha and IL-6 induced by palmitate in 3T3-L1 adipocytes through NF-kappaB and JNK pathway. BES. ; 22(1):32-39.

6. Hong J, Bose M, Ju J, Ryu JH, Chen X, Sang S, et al. (2004): Modulation of arachidonic acid metabolism by curcuminand related beta-diketone derivatives: effects on cytosolicphospholipase $\mathrm{A}(2)$, cyclooxygenases and 5lipoxygenase.Carcinogenesis .25;1671-1679.

7. Ma QL, Yang F, Rosario ER, Ubeda OJ, Beech W, Gant DJ, et al. (2009): Beta-amyloid oligomers induce phosphorylation of tau and inactivation of insulin receptor substrate via c-Jun $\mathrm{N}$ terminal kinase signaling:suppression by omega-3 fatty acids and curcumin. $\mathbf{J}$ Neurosci.; 29:9078-89.

8. Fiala M, Liu PT, Jeffrey A, Rosenthal MJ(2007): Innate immunity and transcription of MGAT-III and Toll-like receptors in Alzheimer's disease patients are improved by bisdemethoxycurcumin. Proc Natl Acad Sci U S A. 104(31): 1284912854.

9. Anand P, Ajaikumar B. Kunnumakkara, Robert A. Newman, and Bharat B. Aggarwal (2007): Bioavailability of Curcumin: Problems and Promises. Mol. Pharmaceutics, 2007, 4 (6), 807-818.

10. Rezq AE, Abdel Aziz MT, and Kumosani TA (2008): PCT/EG2008/000044, Published Patent Pending, WO 2010/057503, Regional phase European Patent Application No. 08878223. 
11. Nesrine S. El Sayed, Lobna A. Kassem, Ola A. Heikal(2009): Promising therapy for Alzheimer's disease targeting angiotensinconverting enzyme and the cyclooxygense- 2 isoform.Drug Discov Ther.; 3(6):307-315.

12. Ishrat T, Hoda MN, Khan MB, Yousuf S, Ahmad M, Khan MM, Ahmad A, Islam F.(2009): Amelioration of cognitive deficits and neurodegeneration by curcumin in rat model of sporadic dementia of Alzheimer's type (SDAT).

Eur

Neuropsychopharmacol.; 19(9):636-47.

13. Lee RH, Pulin AA, Seo MJ, Kota DJ, Ylostalo J, Larson BL, Semprun-Prieto

L, Delafontaine P, Prockop DJ (2009): Intravenous hMSCs improve myocardial infarction in mice because cells embolized in lung are activated to secrete the anti-inflammatory protein TSG-6. Cell Stem Cell; 5:54-63.

14. Alhadlaq A, Mao JJ (2004): Mesenchymal stem cells: isolation and therapeutics. Stem Cells Dev; 13(4):436-48.

15. Jaiswal N, Haynesworth $S$, Caplan A, Bruder S (1997): Osteogenic differentiation of purified, culture-expanded human mesenchymal stem cells in vitro. J Cell Biochem; 64:295-312.

16. Abdel Aziz MT, Atta HM, Samer H , Ahmed HH, Rashed LA, Sabry D,ER Abdel Raouf ER , ALKaffas MA,(2013): Heme oxygenase effect on mesenchymal stem cells action on experimental

Alzheimer's
disease.EXCLI Journal ; 12:778792.

17. Satoh K (1978): Serum Lipid Peroxide in cerebrovascular disorders determined by a new colorimetric method. Clin Chim Acta, 90:37-43.

18. Beutler E.,Duron O., Kelly MB.J.(1963): IImproved method for the determination of blood glutathione.J Lab Clin. Med; 61,882-888.

19. Richmond W (1973): Preparation and Properties of a Cholesterol Oxidase from Nocardia sp. and Its Application to the Enzymatic Assay of Total Cholesterol in Serum. Clin Chem, 19:1350-1356.

20. Banchroft , J.D.; Stevens , A. And Turner, D.R. (1996): theory and practice of histological techniques.fourth ed. Churchil livingstone, new york, london, san francisco, tokyo.

21. Mattson mp (2000): emerging neuroprotective strategies for alzheimer's disease: dietary restriction, telomerase activation, and stem cell therapy. Exp gerontol.;35(4):489-502.

22. Lindvall o, kokaia $z$ (2006): stem cells for the treatment of neurological disorders.nature.; 441(7097):1094-6.

23. Kanazawa $\mathbf{H}$, Fujimoto $\mathbf{Y}$, Teratani T, Iwasaki J, Kasahara $\mathbf{N}$, et al. (2011): Bone MarrowDerived Mesenchymal Stem Cells Ameliorate Hepatic Ischemia Reperfusion Injury in a Rat Model. PLoS ONE 6(4): e19195.

24. Davinelli S, Sapere N, Zella D, Bracale $\mathbf{R}$, Intrieri $\mathbf{M}$, Scapagnini G(2012): Pleiotropic Protective Effects of 
Phytochemicals in Alzheimer's Disease.Oxid Med Cell Longev.: 386527.

25. Mancuso C, Siciliano R, Barone E(2010): Curcumin and Alzheimer disease: this marriage is not to be performed. J Biol Chem.; 286(3):le3.

26. Rubio-Perez JM, Morillas-Ruiz JM (2012): A review: inflammatory process in Alzheimer's disease, role of cytokines.

ScientificWorldJournal.;

2012:756357.

27. Friedman $R$ (2012): Stem cells with curcumin reportedly help heal spinal cord.Neurology today; 10(24): 20.

28. Choi JJ, Yoo SA, Park SJ, Kang YJ , Kim WU, Oh IH, and Cho CS(2008): Mesenchymal stem cells overexpressing interleukin-10 attenuate collagen-induced arthritis in mice. Clin Exp Immunol. ; 153(2): 269-276.

29. Drela K, Siedlecka P, Sarnowska A, Domanska-Janik K (2013): Human mesenchymal stem cells in the treatment of neurological diseases. Acta Neurobiol Exp (Wars). ; 73(1):38-56.

30. Vance JE (2012): Dysregulation of cholesterol balance in the brain ; contribution to neurodegenerative diseases.Dis.Model.Mech.5(6):746 -755 .

31. Searcy James Lucas (2009): Lipid Signaling In Brain Aging And alzheimer's Disease.Pharmacologically

Targetingcholesterol Synthesis, Transportand Metabolism, 15 pages.
32. Tian m, wang $\mathrm{l}$, yu g, liu b, yu li y (2012): curcumin promotes cholesterol efflux from brain through 1xr/rxr-abca1-apoa1 pathway in chronic cerebral hypoperfusion aging-rats. Molecular neurodegeneration, 7(1):s7.

33. Dong S, Zeng Q, Mitchell ES, Xiu J, Duan Y, et al. (2012): Curcumin Enhances Neurogenesis and Cognition in Aged Rats: Implications for Transcriptional Interactions Related to Growth and Synaptic Plasticity. PLoS ONE 7(2): e31211.

34. Pan Ying, Yicun Chen, Qingnan Li, Xiaoyu Yu, Jinzhi Wang and Jinhong Zheng (2013): The Synthesis and Evaluation of Novel Hydroxyl Substituted Chalcone Analogs with in Vitro Anti-Free Radicals Pharmacological Activity and in Vivo Anti-Oxidation Activity in a Free Radical-Injury Alzheimer's Model .Molecules;18:1693-1703.

35. Gustaw-Rothenberg $\mathbf{K}$, Lerner A, Bonda DJ, Lee H, i Zhu X, Perry G, Smith MA(2010): Biomarkers in Alzheimer's disease: past, present and future. Biomark Med.; 4(1): 15-26.

36. EL-DENSHARY ESM, RASHED LA, ELHUSSINY M (2012): Immunosuppressive effects of mesenchymal stem cells versus corticosteroid in experimental model of arthritis. Clin Exp Pharmacol S5:003.

37. Ataie a, sabetkasaei m, haghparast a, hajizadeh moghaddam a, ataie $\mathbf{r}$, nasiraei moghaddam sh. (2010): an investigation of the 
neuroprotective effects of curcumin in a model of homocysteine - induced oxidative stress in the rat's brain. Daru; 18(2):128-36.

38. Ebrahimi $A$ and Lavland $\mathbf{N}$ (2013): Drug delivery using genetically modified mesenchymal stem cells:A promising targeteddelivery method.

Hygeia.J.D.Med., 5 (1): 90-104.
39. Bae JS, Jin HK, Lee JK, Richardson JC, Carter JE.(2013): Bone marrow-derived mesenchymal stem cells contribute to the reduction of amyloid- $\beta$ deposits and the improvement of synaptic transmission in a mouse model of pre-dementia Alzheimer's disease. Curr Alzheimer Res.; 10(5):524-31. 
الطريقة المضادة للالتهابات لعمل كلا من مشتث الكوركومين القابل للأوبان

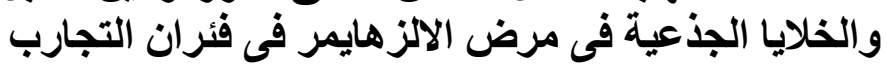

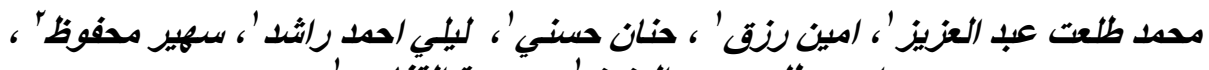

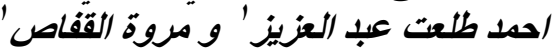

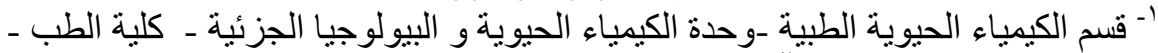

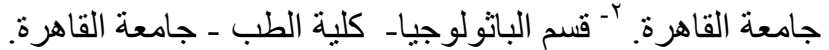

الهدف : تهدف هذه الدر اسة الى تقييم الطرق المحتملة التى يقوم بها كل من مشتق الكركومين و الخلايـا

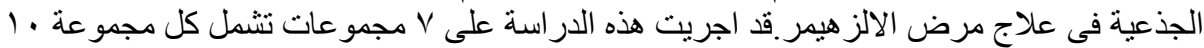

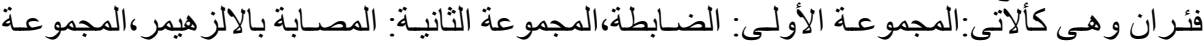

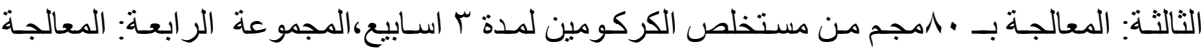

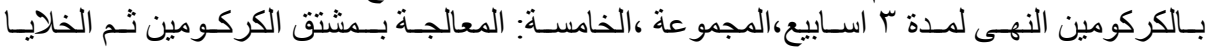

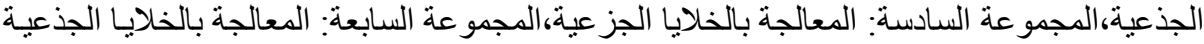

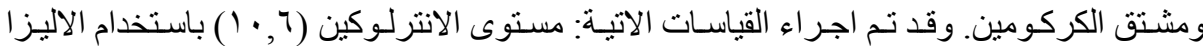

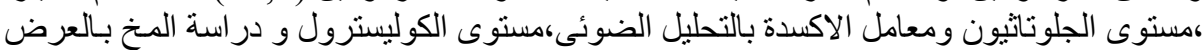

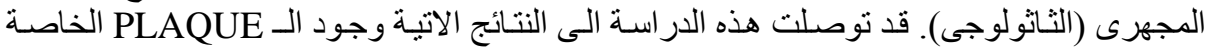

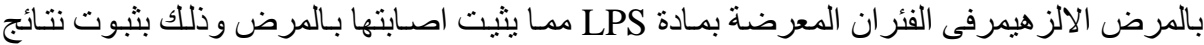

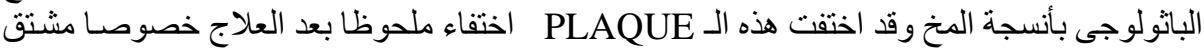

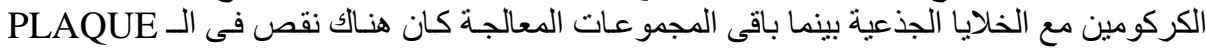

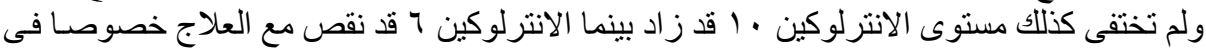

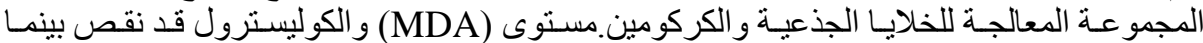

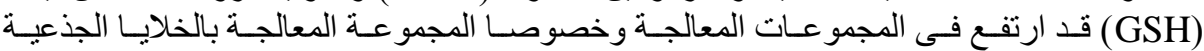

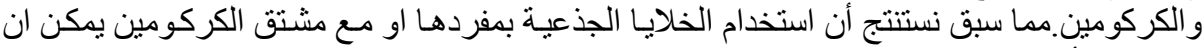

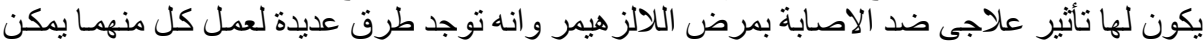

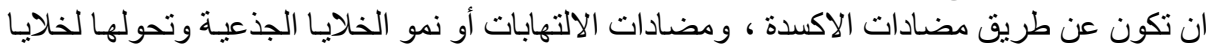

